Original Research Paper

\title{
New Hybrid Block Method with Three Off-Step Points for Solving First Order Ordinary Differential Equations
}

\author{
Ra'ft Abdelrahim, Zurni Omar and John Olusola Kuboye \\ Department of Mathematics, School of Quantitative Sciences, Universiti of Utara Malaysia (UUM), Malaysia
}

\author{
Article history \\ Received: 01-12-2015 \\ Revised: 07-12-2015 \\ Accepted: 12-02-2016 \\ Corresponding Author: \\ John Olusola Kuboye \\ Department of Mathematics, \\ School of Quantitative \\ Sciences, Universiti Utara \\ Malaysia, Malaysia \\ Email: kubbysholly2007@yahoo.com
}

\begin{abstract}
A new single-step hybrid block method with three off-step points for the solution of first order ordinary differential equations is proposed. The strategy employed to develop this method is interpolating the power series approximate solution at $x_{n}$ and off-step points and collocating the derivative of the power at $x_{n+1}$. The class of linear multistep method derived is then simultaneously applied to first order ordinary differential equations together with the associated initial conditions. The numerical results generated are found to be better when compared with the existing methods in terms of error. Besides its excellent performance in term of accuracy, this method also possesses good properties of numerical method such as zerostable, consistent and convergent.
\end{abstract}

Keywords: Hybrid Block, Single-Step, Interpolation, Collocation, Ordinary Differential Equation

\section{Introduction}

We are interested in finding the numerical solution of first order initial value problems of Ordinary Differential Equations (ODEs) in the following form:

$y^{\prime}=f(x, y), \quad y\left(x_{0}\right)=y_{0}, \quad a \leq x \leq b$

Several scholars such as Awoyemi et al. (2007), Badmus and Mishelia (2011), Sunday et al. (2013) developed numerical methods which were implemented in predictor-corrector mode for solving (1). The implementation of numerical method in predictor-corrector approach, however, has some setbacks which include lengthy computational time due to more function evaluations needed per step and computational burden which may affect the accuracy of the method in terms of error (James et al., 2012). In overcoming the setbacks mentioned above, Sagir (2014) developed a three-step block method without predictor where three points with a single off-step point were considered as interpolation points for solving first order ordinary differential equations. The numerical results generated when the method was applied to first order ordinary differential equations are still not encouraging.
Therefore, in this study a single-step block method with three off-step points for solving (1) in order to improve the accuracy of the existing methods is proposed. This paper is divided into four sections; section 1 gives a brief introduction of our work, section 2 explains the derivation of the method, section 3 establishes the properties of the developed block method which include order, error constants, consistency, zerostability and convergence and finally section 4 presents the numerical results derived when the method was tested on first order initial value problems of ODEs.

\section{Derivation of the Method}

Let the power series of the form

$$
y(x)=\sum_{i=0}^{v+m-1} a_{i}\left(\frac{x-x_{n}}{h}\right)^{i}
$$

be considered as the approximate solution to (1) where $v$ represents the number of interpolation points and $m$ is the order of ODE. The derivative (2) is given by:

$$
y^{\prime}(x)=\sum_{i=0}^{v+m-1} \frac{i}{h} a_{i}\left(\frac{x-x_{n}}{h}\right)^{i-1}
$$


Interpolating (1) at $x=x_{n+i}, i=0, \frac{1}{4}, \frac{1}{2}, \frac{3}{4}$ and collocating

(3) at $x=x_{n+1}$ produces equations which can be presented in the following matrix form:

$$
\left[\begin{array}{ccccc}
1 & 0 & 0 & 0 & 0 \\
1 & \frac{1}{4} & \frac{1}{16} & \frac{1}{64} & \frac{1}{256} \\
1 & \frac{1}{2} & \frac{1}{4} & \frac{1}{8} & \frac{1}{16} \\
1 & \frac{3}{4} & \frac{9}{16} & \frac{27}{64} & \frac{81}{256} \\
0 & \frac{1}{h} & \frac{2}{h} & \frac{3}{h} & \frac{4}{h}
\end{array}\right]\left[\begin{array}{l}
a_{0} \\
a_{1} \\
a_{2} \\
a_{3} \\
a_{4}
\end{array}\right]=\left[\begin{array}{c}
y_{n} \\
y_{n+\frac{1}{4}} \\
y_{n+\frac{1}{2}} \\
y_{n+\frac{3}{4}} \\
f_{n+1}
\end{array}\right]
$$

Gaussian elimination method is then applied to determine the values of the unknown variables $a_{j}, j=$ $0(1) 4$, given as below:

$$
\begin{aligned}
& a_{0}=y_{n} \\
& a_{1}=\left(-\frac{616}{75} y_{n}+\frac{256}{75} y_{n+\frac{3}{4}}+\frac{384}{25} y_{n+\frac{1}{4}}-\frac{264}{25} y_{n+\frac{1}{2}}-\frac{3 h}{25} f_{n+1}\right) \\
& a_{2}=\left(\frac{1684}{75} y_{n}-\frac{1744}{75} y_{n+\frac{3}{4}}-\frac{16161}{25} y_{n+\frac{1}{4}}+\frac{1636}{25} y_{n+\frac{1}{2}}+\frac{22 h}{25} f_{n+1}\right) \\
& a_{3}=\left(-\frac{1856}{75} y_{n}+\frac{3296}{75} y_{n+\frac{3}{4}}+\frac{2144}{25} y_{n+\frac{1}{4}}-\frac{2624}{25} y_{n+\frac{1}{2}}-\frac{48 h}{25} f_{n+1}\right) \\
& a_{4}=\left(\frac{704}{75} y_{n}-\frac{1664}{75} y_{n+\frac{3}{4}}-\frac{896}{25} y_{n+\frac{1}{4}}+\frac{1216}{25} y_{n+\frac{1}{2}}+\frac{32 h}{25} f_{n+1}\right)
\end{aligned}
$$

Substituting the values of $a_{s}^{\prime}$ into (2) to give a continuous implicit scheme of the form:

$y(x)$

$$
\begin{aligned}
& =y_{n}+\frac{\left(x-x_{n}\right)^{4}}{h^{4}}\left(\frac{704}{75} y_{n}-\frac{1664}{75} y_{n+\frac{3}{4}}-\frac{896}{75} y_{n+\frac{1}{4}}+\frac{1216}{25} y_{n+\frac{1}{2}}+\frac{32 h}{25} f_{n+1}\right) \\
& +\frac{\left(x-x_{n}\right)^{2}}{h^{2}}\left(\frac{1684}{75} y_{n}-\frac{1744}{75} y_{n+\frac{3}{4}}-\frac{1616}{25} y_{n+\frac{1}{4}}+\frac{1636}{25} y_{n+\frac{1}{2}}+\frac{22 h}{25} f_{n+1}\right) \\
& -\frac{\left(x-x_{n}\right)^{3}}{h^{3}}\left(\frac{1856}{75} y_{n}-\frac{3296}{75} y_{n+\frac{3}{4}}-\frac{2144}{25} y_{n+\frac{1}{4}}+\frac{2624}{25} y_{n+\frac{1}{2}}+\frac{48 h}{25} f_{n+1}\right) \\
& -\frac{\left(x-x_{n}\right)}{h}\left(\frac{616}{75} y_{n}-\frac{256}{75} y_{n+\frac{3}{4}}-\frac{384}{25} y_{n+\frac{1}{4}}+\frac{264}{25} y_{n+\frac{1}{2}}+\frac{3 h}{25} f_{n+1}\right)
\end{aligned}
$$

Evaluating Equation 5 at the non-interpolating point $x$ $=x_{n+1}$ yields:

$$
y_{n+1}+\frac{3}{25} y_{n}-\frac{48}{25} y_{n+\frac{3}{4}}-\frac{16}{25} y_{n+\frac{1}{4}}+\frac{36}{25} y_{n+\frac{1}{2}}=\frac{3 h}{25} f_{n+1}
$$

Equation 5 is then differentiated and evaluated at the off-grid points $\left(x=x_{n+i, i=\frac{1}{4}, \frac{1}{2}, \frac{3}{4}}\right)$ to produce:

$$
\begin{aligned}
& f_{n+\frac{1}{4}}=\frac{-1}{25 h}\left(26 y_{n}+34 y_{n+\frac{3}{4}}+78 y_{n+\frac{1}{4}}-138 y_{n+\frac{1}{2}}\right)+\frac{1}{25} f_{n+1 .} . \\
& f_{n+\frac{1}{2}}=\frac{1}{75 h}\left(28 y_{n}+152 y_{n+\frac{2}{3}}+-216 y_{n+\frac{1}{4}}+36 y_{n+\frac{1}{2}}\right)-\frac{1}{25} f_{n+1 .} . \\
& f_{n+\frac{3}{4}}=\frac{-1}{75 h}\left(34 y_{n}-394 y_{n+\frac{3}{4}}-198 y_{n+\frac{1}{4}}+558 y_{n+\frac{1}{2}}\right)+\frac{3}{25} f_{n+1}
\end{aligned}
$$

Combining Equation 6 and 7 give the following equations presented in a matrix form as below:

$$
R\left[\begin{array}{c}
y_{n+\frac{1}{4}} \\
y_{n+\frac{1}{2}} \\
y_{n+\frac{3}{4}} \\
y_{n+1}
\end{array}\right]=T\left[\begin{array}{c}
y_{n-3} \\
y_{n-2} \\
y_{n-1} \\
y_{n}
\end{array}\right]+U\left[\begin{array}{c}
f_{n+\frac{1}{4}} \\
f_{n+\frac{1}{2}} \\
f_{n+\frac{3}{4}} \\
f_{n+1}
\end{array}\right]
$$

where:

$$
R=\left[\begin{array}{cccc}
\frac{-16}{25} & \frac{36}{25} & \frac{-48}{25} & 1 \\
\frac{-78}{(25 h)} & \frac{138}{(25 h)} & \frac{-34}{(25 h)} & 0 \\
\frac{-72}{(25 h)} & \frac{12}{(25 h)} & \frac{152}{(75 h)} & 0 \\
\frac{66}{(25 h)} & \frac{-186}{(25 h)} & \frac{394}{(75 h)} & 0
\end{array}\right], T=\left[\begin{array}{cccc}
0 & 0 & 0 & \frac{-3}{(25 h)} \\
0 & 0 & 0 & \frac{26}{(25 h)} \\
0 & 0 & 0 & \frac{-28}{(75 h)} \\
0 & 0 & 0 & \frac{34}{(75 h)}
\end{array}\right]
$$

and:

$$
U=\left[\begin{array}{cccc}
0 & 0 & 0 & \frac{3 h}{25} \\
1 & 0 & 0 & \frac{-1}{25} \\
0 & 1 & 0 & \frac{1}{25} \\
0 & 0 & 1 & \frac{-3}{25}
\end{array}\right]
$$

Multiplying (8) with

$\left[\begin{array}{cccc}\frac{-16}{25} & \frac{36}{25} & \frac{-48}{25} & 1 \\ \frac{-78}{(25 h)} & \frac{138}{(25 h)} & \frac{-34}{(25 h)} & 0 \\ \frac{-72}{(25 h)} & \frac{12}{(25 h)} & \frac{152}{(75 h)} & 0 \\ \frac{66}{(25 h)} & \frac{-186}{(25 h)} & \frac{394}{(75 h)} & 0\end{array}\right]^{-1}$

We will get a block method of the form: 


$$
V\left[\begin{array}{c}
y_{n+\frac{1}{4}} \\
y_{n+\frac{1}{2}} \\
y_{n+\frac{3}{4}} \\
y_{n+1}
\end{array}\right]=W\left[\begin{array}{c}
y_{n-3} \\
y_{n-2} \\
y_{n-1} \\
y_{n}
\end{array}\right]+Y\left[\begin{array}{c}
f_{n+\frac{1}{4}} \\
f_{n+\frac{1}{2}} \\
f_{n+\frac{3}{4}} \\
f_{n+1}
\end{array}\right]
$$

For:

$$
\begin{aligned}
& V=\left[\begin{array}{llll}
1 & 0 & 0 & 0 \\
0 & 1 & 0 & 0 \\
0 & 0 & 1 & 0 \\
0 & 0 & 0 & 1
\end{array}\right], W=\left[\begin{array}{llll}
0 & 0 & 0 & 1 \\
0 & 0 & 0 & 1 \\
0 & 0 & 0 & 1 \\
0 & 0 & 0 & 1
\end{array}\right] \\
& Y=\left[\begin{array}{cccc}
\frac{55 h}{96} & \frac{-59 h}{96} & \frac{37}{96} & \frac{-3 h}{32} \\
\frac{2 h}{3} & \frac{-5 h}{12} & \frac{h}{3} & \frac{-h}{12} \\
\frac{21 h}{32} & \frac{-9 h}{32} & \frac{15 h}{32} & \frac{-3 h}{32} \\
\frac{2 h}{3} & \frac{-h}{3} & \frac{2 h}{3} & 0
\end{array}\right]
\end{aligned}
$$

which can also be represented as:

$$
\begin{aligned}
& y_{n+\frac{1}{4}}=y_{n}-\frac{3 \mathrm{~h}}{32} f_{n+1}+\frac{37 \mathrm{~h}}{96} f_{n+\frac{3}{4}}+\frac{55 \mathrm{~h}}{96} f_{n+\frac{1}{4}}-\frac{59 \mathrm{~h}}{96} f_{n+\frac{1}{2}} \\
& y_{n+\frac{1}{2}}=y_{n}-\frac{\mathrm{h}}{12} f_{n+1}+\frac{\mathrm{h}}{3} f_{n+\frac{3}{4}}+\frac{2 \mathrm{~h}}{3} f_{n+\frac{1}{4}}-\frac{5 \mathrm{~h}}{12} f_{n+\frac{1}{2}} \\
& y_{n+\frac{3}{4}}=y_{n}-\frac{3 \mathrm{~h}}{32} f_{n+1}+\frac{15 \mathrm{~h}}{32} f_{n+\frac{3}{4}}+\frac{21 \mathrm{~h}}{32} f_{n+\frac{1}{4}}-\frac{9 \mathrm{~h}}{32} f_{n+\frac{1}{2}} . \\
& y_{n+1}=y_{n}+\frac{2 \mathrm{~h}}{3} f_{n+\frac{3}{4}}+\frac{2 \mathrm{~h}}{3} f_{n+\frac{1}{4}}-\frac{\mathrm{h}}{3} f_{n+\frac{1}{2}}
\end{aligned}
$$

\section{Properties of the Method}

Based on the definition given by Lambert (1973) and Henrici (1962), the order of the developed method is $[4,4,4,4]^{T}$ with vector error constant:

$$
\left(\bar{c}_{5}\right)=\left[3.4044 e^{(-4)}, 3.1467 e^{(-4)}, 3.2959 e^{(-4)}, 3.0382 e^{(-4)}\right]^{T}
$$

The first characteristic polynomial of (8) is given by:

$$
\begin{aligned}
& \rho(\tau)=\left\lfloor\tau\left|\left[\left[\begin{array}{llll}
1 & 0 & 0 & 0 \\
0 & 1 & 0 & 0 \\
0 & 0 & 1 & 0 \\
0 & 0 & 0 & 1
\end{array}\right]\right]-\left[\left[\begin{array}{llll}
0 & 0 & 0 & 1 \\
0 & 0 & 0 & 1 \\
0 & 0 & 0 & 1 \\
0 & 0 & 0 & 1
\end{array}\right]\right\rfloor\right|\right. \\
& =\tau^{3}(\tau-1)
\end{aligned}
$$

which satisfies the zero-stability condition stated by Lambert (1973) that is $\left|\tau_{j}\right| \leq 1$ and when $\left|\tau_{j}\right|=1$, the multiplicity must not exceed 1 .

Since the order of method (8) is greater than 1 , it is therefore consistent. Furthermore, the developed method is also convergent because it is zero stable and consistent.

\section{Numerical Examples}

The following problems available in the previous literatures were solved in order to compare the performance of the new method with existing ones

Problem 1: $y^{\prime}=x y, y(0)=1, h=0.1$

$$
\text { Exact solution: } y=e^{\frac{x^{2}}{2}}
$$

The numerical results of our method and the method developed by Odekunle et al. (2012) for solving Problem 1 are tabulated in Table 1 below.

Problem 2: $y^{\prime}=0.2 y, y(0)=10000, h=0.1$

Exact solution: $y=1000 e^{\frac{x^{2}}{2}}$

The above problem is spatial case of differential equation of growth model which describes the growth rate of bacteria in a colony every hour by assuming that the bacteria grows continuously without any restriction. This problem was solved by Sagir (2014) using threestep hybrid block method of order four. The numerical results comparing our method with Sagir (2014) for solving Problem 2 are depicted in Table 2 below.

Table 1. Comparison of the new method with Odekunle et al. (2012) for solving Problem 1

\begin{tabular}{lllll}
\hline$x$ & Exact solution & Computed solution & Error in new method & Error in Odekunle et al. (2012) \\
\hline 0.1 & 1.005012520859401000 & 1.005012521195772800 & $3.363718 e^{-10}$ & $2398 e^{-7}$ \\
0.2 & 1.020201340026755800 & 1.020201341302355800 & $1.275600 e^{-9}$ & $1.6913 e^{-7}$ \\
0.3 & 1.046027859908716900 & 1.046027862194445400 & $2.285728 e^{-9}$ & $8.7243 e^{-7}$ \\
0.4 & 1.083287067674958600 & 1.083287069062809600 & $1.387851 e^{-9}$ & $3.0098 e^{-6}$ \\
0.5 & 1.133148453066826300 & 1.133148447836481300 & $5.230345 e^{-9}$ & $1.7466 e^{-6}$ \\
0.6 & 1.197217363121810200 & 1.197217339115218500 & $2.400659 e^{-8}$ & $4.1710 e^{-6}$ \\
0.7 & 1.277621313204886600 & 1.277621247849590700 & $6.535530 e^{-8}$ & $9.6465 e^{-6}$ \\
0.8 & 1.377127764335957000 & 1.377127618495140200 & $1.458408 e^{-7}$ & $6.7989 e^{-6}$ \\
0.9 & 1.499302500056766800 & 1.499302208459504200 & $2.915973 e^{-7}$ & $5.436669 e^{-7}$ \\
1.0 & 1.648721270700128000 & 1.648720727033248700 & & $2.6575 e^{-5}$ \\
\hline
\end{tabular}


Table 2. Comparison of the new method with Sagir (2014) for solving Problem 2

\begin{tabular}{|c|c|c|c|c|}
\hline$x$ & Exact solution & Computed solution & Error in new method & Error in Sagir (2014) \\
\hline 0.1 & 1020.201340 & 1020.201340 & $00000000 \mathrm{e}+00$ & $00000000 \mathrm{e}+00$ \\
\hline 0.2 & 1040.810774 & 1040.810774 & $00000000 \mathrm{e}+00$ & $00000000 \mathrm{e}+00$ \\
\hline 0.3 & 1061.836547 & 1061.836547 & $00000000 \mathrm{e}+00$ & $00000000 \mathrm{e}+00$ \\
\hline 0.4 & 1083.287068 & 1083.287068 & $00000000 \mathrm{e}+00$ & $00000000 \mathrm{e}+00$ \\
\hline 0.5 & 1105.170918 & 1105.170919 & $1.000000000 e^{-6}$ & $00000000 \mathrm{e}+00$ \\
\hline 0.6 & 1127.496852 & 1127.496852 & $00000000 \mathrm{e}+00$ & $00000000 \mathrm{e}+00$ \\
\hline 0.7 & 1150.273799 & 1150.273710 & $1.000000000 e^{-6}$ & $1.000000000 e^{-6}$ \\
\hline 0.8 & 1173.510871 & 1173.510872 & $1.000000000 e^{-6}$ & $4.800000000 e^{-5}$ \\
\hline 0.9 & 1197.217363 & 1197.217364 & $1.000000000 e^{-6}$ & $5.100000000 e^{-5}$ \\
\hline 1.0 & 1221.402758 & 1221.402759 & $1.000000000 e^{-6}$ & $6.600000000 e^{-5}$ \\
\hline
\end{tabular}

\section{Discussion of the Results}

In Table 1, the results produced from the new method for solving Problem 1 have better accuracy when compared with the results in Odekunle et al. (2012). It can also be seen in Table 2 that the new method is more efficient in terms of error than Sagir (2014).

\section{Conclusion}

A new one-step hybrid block method of order four for solving first ODEs has been developed in this study. The numerical solutions are produced with less computational efforts when compared with non-block method. The new method is consistent, zero-stable and convergent. In term of accuracy, this method claims superiority over the existing methods. Hence, the new developed method should be opted to solve first order initial value problems of ODEs directly.

\section{Acknowledgement}

Thanks to the Universiti Utara Malaysia for the financial assistance rendered in publishing this paper.

\section{Author's Contribution}

All authors equally contributed in this work.

\section{Conflict of Interest}

The authors declare that there is no conflict of interests regarding the publication of this paper.

\section{References}

Awoyemi, D.O., R.A. Ademiluyi and E. Amusegham, 2007. Off-grid points exploitation in the development of more accurate collocation method for solving ODEs. J. Nigerian Assoc. Math. Phys., 12: 379-386.

Badmus, A.M. and D.W. Mishehia, 2011. Some uniform order block methods for the solution of first ordinary differential equation. J. Nigerian Assoc. Math. Phys., 19: 149-154.

Henrici, P., 1962. Discrete variable methods in ordinary differential equations. 1st Edn., Willey, New York, pp: 407.

James, A.A., A.O. Adesanya and J. Sunday, 2012. uniform order continuous block hybrid method for the solution of first order ordinary differential equations. IOSR J. Math., 3: 8-14.

Lambert, J.D., 1973. Computational Methods in Ordinary Differential Equations. 1st Edn., John Willey and Sons, New York, ISBN-10: 0471511943, pp: 278.

Odekunle, M.R., A.O. Adesanya and J. Sunday, 2012. A new block integrator for the solution of initial value problems of first-order ordinary differential equations. Int. J. Pure Applied Sci. Technol., 11: 92-100.

Sagir, A.M., 2014. Numerical treatment of block method for the solution of ordinary differential equations. Int. J. Math. Comput. Phys. Quantum Eng., 8: 81-81.

Sunday, J., M.R. Odekunle and A.O. Adeyanya, 2013. Order six block integrator for the solution of firstorder ordinary differential equations. Int. J. Math. Soft Comput., 3: 87-96. 